\title{
28 Research Square \\ Epidemiology and Clinical Features of Anterior Uveitis: a Prospective Study
}

\section{Wei-Yu Chiang}

Chang Gung Memorial Hospital Kaohsiung Branch https://orcid.org/0000-0002-0791-8303

\section{Shih-Chou Chen}

Kaohsiung Veterans General Hospital

\section{Shwu-Jiuan Sheu}

Kaohsiung Medical University Hospital: Kaohsiung Medical University Chung Ho Memorial Hospital

Hsi-Kung Kuo ( $\nabla$ hsikung@cgmh.org.tw )

Kaohsiung Medical Center: Chang Gung Memorial Hospital Kaohsiung Branch

\section{Research Article}

Keywords: anterior uveitis, CMV iritis, herpetic anterior uveitis, HLA-B27, prevalence

Posted Date: January 4th, 2022

DOI: https://doi.org/10.21203/rs.3.rs-1159747/v1

License: (c) (1) This work is licensed under a Creative Commons Attribution 4.0 International License. Read Full License 


\section{Abstract}

Purpose: To report the pattern, epidemiology, and clinical features of anterior uveitis (AU) in southern Taiwan

Methods: A prospective case series was performed to recruit patients with AU at two medical centers (Kaohsiung Chang Gung Memorial Hospital and Kaohsiung Veterans General Hospital) in southern Taiwan from December 1, 2018 to March 31, 2020. These patients enrolled were reviewed for clinical diagnoses, ocular presentations, and laboratory data, including aqueous polymerase chain reaction tests.

Results: A total of 112 patients were included. The most common clinical diagnoses were idiopathic AU (37.5\%), HLA-B27-associated acute AU (25.0\%), and herpetic AU (18.8\%); among herpetic etiology, cytomegalovirus (CMV) was the most common pathogen. Hypertensive AU was associated with older age, more males, and greater numbers of corneal edema, and fewer iris posterior synechiae. Compared with HLA-B27-associated acute AU, CMV AU was associated with older age, high intraocular pressure, more keratic precipitates, greater iris atrophy, more pseudophakia, and fewer pupil posterior synechiae.

Conclusion: This prospective study identified the epidemiological and clinical features of AU in southern Taiwan. The most common etiologies were idiopathic AU, HLA-B27-associated acute AU, and herpetic (most CMV) AU.

\section{Introduction}

Uveitis, also referred to as intraocular inflammation, is a complex inflammatory process, primarily involving the uveal tract, with or without the involvement of the adjacent intraocular structures. Uveitis is a major cause of ocular morbidity, contributing to $5-10 \%$ of visual impairment worldwide [1].

Approximately $35 \%$ of patients with uveitis suffer from significant vision loss due to legal blindness [2]. Moreover, most affected individuals are at their working age (20-65 years), resulting in significant socioeconomic impacts [3]. The differential diagnosis of uveitis varies and is influenced by several factors, such as genetic, ethnic, geographic, environmental, nutritional, and socioeconomic factors [4]. Infections are a leading cause of uveitis in developing countries, whereas idiopathic uveitis is the leading cause in developed countries [5]. Determining the proper underlying etiology is challenging $[5,6]$.

Uveitis is subclassified as granulomatous or non-granulomatous anterior, intermediate, posterior or panuveitis based on the anatomical involvement of the eye. Anterior uveitis (AU) is the most common type of uveitis, with varying incidences reported in the literature worldwide [7-11]. Jakob et al reported anterior uveitis, intermediate uveitis, posterior uveitis, and panuveitis in $45.5 \%, 22.9 \%, 13.5 \%$, and $6.2 \%$ of patients, respectively. The remaining $12.0 \%$ of cases showed extrauveal manifestations (scleritis, episcleritis, keratitis, optic neuritis, myositis, and orbital inflammation) [12]. In a 22-year period from the UK, the anatomical types observed were anterior (46\%), intermediate (11.1\%), posterior $(21.8 \%)$, and panuveitis (21.1\%) [13]. Based on the Taiwan National Health Insurance Research Database, Hwang et al 
reported the uveitis epidemiology study: AU was found in $77.7 \%$ of the incident cases; $15.2 \%, 6.7 \%$, and $0.4 \%$ were cases of panuveitis, posterior uveitis, and intermediate uveitis, respectively [9].

AU can be benign, but can often lead to severe morbidity if not treated appropriately $[6,14]$. AU can be treated without long-term sequelae if diagnosed and treated on time. There are several known possible etiologies of AU [15]. Human leukocyte antigen (HLA)-B27 associated acute AU is a distinct clinical entity, which accounts for 6-13\% of all AU in Asia [16]. Non-HLA-B27-associated acute AU consists of various etiologies, such as Posner-Schlossman syndrome (PSS), Fuchs' heterochromic iridocyclitis (FHI), herpetic $A U$, juvenile idiopathic uveitis (JIA), or other panuveitis initially presenting as AU. Among patients with non-HLA-B27-associated acute AU, some may present with ocular hypertension, such as PSS, FHI, or herpetic AU. Herpetic AU caused by herpesviridae, including herpes simplex virus (HSV), varicella-zoster virus (VZV), cytomegalovirus (CMV), and Epstein-Barr virus (EBV), has specific manifestations. Our previous retrospective study which included patients with HLA-B27 negative AU with increased intraocular pressure or corneal edema, indicated that $41.1 \%$ were herpesviridae-positive and CMV was the most common etiology [17].

In Eastern Asia, few prospective studies have clarified the etiology of AU. This study aimed to investigate the current etiologies and clinical features of AU in Taiwan using a prospective method.

\section{Methods}

\section{Patients}

This prospective study was conducted in Kaohsiung city in southern Taiwan from December 2018 to March 2020. Patients were enrolled from two medical centers (Kaohsiung Chang Gung Memorial Hospital and Kaohsiung Veterans General Hospital) by four uveitis specialists (Dr. Shwu-Jiuan Sheu, Dr. Hsi-Kung Kuo, Dr. Shih-Chou Chen, and Dr. Wei-Yu Chiang). The inclusion criteria were patients with the clinical manifestations of $\mathrm{AU}$, which was diagnosed based on history, ocular examination, laboratory data, and image data. However, patients with uveitis other than AU were excluded. The study adhered to the Declaration of Helsinki and was approved by the Institutional Review Board of the Chang Gung Memorial Hospital (study reference number: 201702154A3). Informed consent was obtained from all participants.

\section{Protocol}

The protocol included a 2-step examination; the first step included collecting demographics, clinical presentations, and laboratory tests; the second step included an aqueous humor polymerase chain reaction (PCR) test. The detailed collected items included age, sex, history of first episode or not, symptom duration, and acute course, ocular examination of one or both affected eyes, intraocular pressure (IOP), keratic precipitates (KP), corneal edema, hypopyon, iris atrophy, posterior synechiae (PS), and intraocular lens (IOL). The acute course was defined as a sudden onset and limited duration of $\leq 3$ months. Laboratory tests included complete blood count, HLA-B27, C-reactive protein, erythrocyte sedimentation rate, antinuclear antibody, rheumatoid factor, rapid plasma regain, Treponema pallidum 
hemagglutination, and chest and sacroiliac X-ray. An aqueous PCR test was designed for CMV, HSV, VZV, and EBV. If the first step confirmed the diagnosis of HLA-B27-associated acute AU with HLA-B27 positivity and its corresponding presentations, the PCR test could be neglected. If HLA-B27-associated acute AU was absent, the patient would undergo an aqueous PCR examination.

\section{Aqueous humor PCR}

If clinically indicated, patients with AU underwent PCR for aqueous humor DNA extraction and amplification. Anterior chamber paracentesis with a $27 \#$-gauge needle was performed using an aseptic technique with the aid of a microscope. The details of the sample processing and primer information are described in our previous study [17].

\section{Statistics}

In the descriptive analysis, categorical data were shown as numbers and percentages and continuous variables were expressed as mean \pm standard deviations (SDs). Comparisons were performed using Student's t-test for continuous variables and Chi-square test for categorical factors. In the multivariate analyses, stepwise logistic regression analysis was used to identify significant independent predictors for differentiating HLA-B27-associated and CMV AU. Statistical significance was defined as a two-tailed $p$ value of $<0.05$.

\section{Results}

A total of 112 patients were enrolled in this study. The final diagnoses are shown in Table 1. The etiologies were idiopathic acute and chronic AU (37.5\%), HLA-B27-associated acute AU (25.0\%), herpetic AU (18.8\%), PSS (11.6\%), FHI (3.6\%), JIA (2.7\%), and Behcet's disease (0.9\%). The diagnoses were based on the physicians' clinical impressions. The clinical manifestations of PSS included recurrent unilateral, mild, acute non-granulomatous anterior uveitis with markedly elevated IOP, corneal edema, KPs, lowgrade cell, and vague symptoms. The clinical manifestations of FHI included recurrent unilateral anterior uveitis with small KPs, presence of heterochromia, lack of synechiae, and lack of symptoms. All patients with suspected PSS and FHI underwent aqueous PCR. If PCR was positive, then these cases were classified as herpetic AU; otherwise, they were classified as initial PSS or FHI diagnoses. The demographics and clinical manifestations of all the participants are listed in Table 2.

\section{Table 1. The clinical diagnosis of anterior uveitis}

The most common etiologies were idiopathic AU, HLA-B27-associated AU, and herpetic AU. 


\begin{tabular}{|ll|}
\hline Idiopathic AU (acute and chronic) & $37.5 \%, 42 / 112$ \\
\hline HLA-B27-associated acute AU & $25.0 \%, 28 / 112$ \\
\hline Herpetic AU (CMV, HSV, VZV, EBV) & $18.8 \%, 21 / 112$ \\
\hline Posner-Schlossman syndrome (PSS) & $11.6 \%, 13 / 112$ \\
\hline Fuchs heterochromic iridocyclitis (FHI) & $3.6 \%, 4 / 112$ \\
\hline Juvenile idiopathic uveitis (JIA) & $2.7 \%, 3 / 112$ \\
\hline Behcet disease & $0.9 \%, 1 / 112$ \\
\hline
\end{tabular}

AU: anterior uveitis

The data of the ocular hypertension $(I O P>21 \mathrm{mmHg}$ ) and non-ocular hypertension (IOP $\leq 21 \mathrm{mmHg})$ groups are presented in Table 2. The mean IOP was 36.2 vs. $12.7 \mathrm{mmHg}(\mathrm{p}<0.001)$ and the IOP difference from the fellow eye was 21.6 vs. $-1.2 \mathrm{mmHg}(p<0.001)$. The ocular hypertension group demonstrated older age ( 55.2 vs. 46.6 years, $p=0.002)$, more male $(76.7 \%$ vs. $50.0 \%, p=0.012)$, longer duration (20.4 vs. 13.1 days, $p=0.099)$, more corneal edema ( $53.3 \%$ vs. $24.4 \%, p=0.004)$, less iris PS $(0.0 \%$ vs. $19.5 \%, p=0.009)$ and less HLA-B27 positive $(10.3 \%$ vs. $33.8 \%, p=0.016)$ than the non-ocular hypertension group.

Table 2. Demographics and clinical manifestations of all participants and the comparison between ocular hypertension $(I O P>21)$ and non-ocular hypertension (IOP $\leqq 21)$ with $p$ values

The ocular hypertension group demonstrated older age, more males, longer duration, more corneal edema, less iris PS and less HLA-B27 positivity. 


\begin{tabular}{|lllll|}
\hline & Total & IOP>21 group & IOP $\leqq 21$ group & p value \\
\hline Case No. & 112 & 30 & 82 & \\
\hline IOP (mmHg) & $19.9 \pm 12.6(6.6-55.7)$ & 36.2 & 12.7 & $<0.001^{\star}$ \\
\hline IOP difference $(\mathrm{mmHg})$ & $5.8 \pm 12.5(-11.2-42.4)$ & 21.6 & -1.2 & $<0.001^{\star}$ \\
\hline Age (years) & $48.9 \pm 16.1(9-83)$ & 55.2 & 46.6 & $0.002^{\star}$ \\
\hline Male & $48(57.1 \%)$ & $76.7 \%$ & $50.0 \%$ & $0.012^{\star}$ \\
\hline First episode & $46(41.1 \%)$ & $30.0 \%$ & $45.1 \%$ & 0.150 \\
\hline Acute course & $86(77.5 \%)$ & $73.3 \%$ & $79.0 \%$ & 0.525 \\
\hline Duration (days) & $15.0 \pm 18.0(1-120)$ & 20.4 & 13.1 & 0.099 \\
\hline OU & $14(12.5 \%)$ & $3.3 \%$ & $15.9 \%$ & 0.076 \\
\hline KP & $81(72.3 \%)$ & $83.3 \%$ & $68.3 \%$ & 0.115 \\
\hline K edema & $36(32.1 \%)$ & $53.3 \%$ & $24.4 \%$ & $0.004^{\star}$ \\
\hline Hypopyon & $6(5.4 \%)$ & $0.0 \%$ & $7.3 \%$ & 0.128 \\
\hline PS & $16(14.3 \%)$ & $0.0 \%$ & $19.5 \%$ & $0.00{ }^{\star}$ \\
\hline Iris atrophy & $14(12.5 \%)$ & $20.0 \%$ & $9.8 \%$ & 0.147 \\
\hline Pseudophakia & $23(20.5 \%)$ & $20.7 \%$ & $20.0 \%$ & 0.932 \\
\hline HLA-B27 & $30(27.5 \%)$ & $10.3 \%$ & $33.8 \%$ & $0.016^{\star}$ \\
\hline PCR(+) & $21 / 61(34.4 \%)$ & $30.0 \%$ & $14.6 \%$ & 0.065 \\
\hline CMV & $17 / 21(81.0 \%)$ & $34.6 \%$ & $22.9 \%$ & 0.311 \\
\hline HSV & $1 / 21(9.5 \%)$ & $0.0 \%$ & $2.9 \%$ & 0.385 \\
\hline VZV & $0.0 \%$ & $3.3 \%$ & 0.367 \\
\hline EBV & $0.0 \%$ & $7.1 \%$ & 0.182 \\
\hline
\end{tabular}

IOP: intraocular pressure, KP: keratic precipitates, PS: posterior synechiae, PCR: polymerase chain reaction

The HLA-B27-associated acute AU (28 cases) and CMV related AU (17 cases) groups are compared in Table 3. With statistical significance, the HLA-B27-associated acute AU group showed younger age (38.8 vs. 59.2 years, $p<0.001)$, more first episodes $(64.3 \%$ vs. $11.8 \%, p=0.001)$, more acute courses $(96.4 \%$ vs. $58.8 \%, p=0.003$ ), shorter symptom durations ( 9.6 vs. 26.2 days, $p=0.032$ ), lower IOP (10.2 vs. 26.3 $\mathrm{mmHg}, \mathrm{p}<0.001)$, less ocular hypertension ( $0.0 \%$ vs. $47.1 \%, p<0.001)$, lower IOP difference from the fellow eye (-4.2 vs. $11.4 \mathrm{mmHg}, \mathrm{p}<0.001)$, less corneal $\mathrm{KPs}(60.7 \%$ vs. $88.2 \%, p=0.048)$, more iris PS 
( $35.7 \%$ vs. $0.0 \%, p=0.007)$, less iris atrophy $(0.0 \%$ vs. $41.2 \%, p<0.001)$, and less pseudophakias $(7.1 \%$ vs. $35.3 \%, p=0.039)$. Among these factors, the multivariate analysis identified the IOP $(p<0.001)$, IOP difference $(p<0.001)$, iris atrophy $(p=0.007)$, age $(p=0.020)$, and pseudophakia $(p=0.029)$ as significant indicators for distinguishing HLA-B27-associated acute AU and CMV related AU.

\section{Table 3. The comparison between HLA-B27-associated acute AU and CMV AU}

The HLA-B27-associated acute AU group showed younger age, more first episode, more acute course, shorter duration, lower IOP, less ocular hypertension, lower IOP difference from the fellow eye, less corneal KP, more iris PS, less iris atrophy, and less pseudophakia.

\begin{tabular}{|llll|}
\hline & HLA-B27 & CMV & p value \\
\hline Case No. & 28 & 17 & \\
\hline IOP & 10.2 & 26.3 & $<0.001^{\star}$ \\
\hline IOP $>21$ & $0.0 \%$ & $47.1 \%$ & $<0.001^{\star}$ \\
\hline IOP difference & -4.2 & 11.4 & $<0.001^{\star}$ \\
\hline Age & 38.8 & 59.2 & $<0.001^{\star}$ \\
\hline Male & $46.4 \%$ & $70.6 \%$ & 0.114 \\
\hline First episode & $64.3 \%$ & $11.8 \%$ & $0.001^{\star}$ \\
\hline Acute course & $96.4 \%$ & $58.8 \%$ & $0.003^{\star}$ \\
\hline Duration & 9.6 & 26.2 & $0.032^{\star}$ \\
\hline OU & $3.6 \%$ & $0.0 \%$ & 1.000 \\
\hline KP & $60.7 \%$ & $88.2 \%$ & $0.048^{\star}$ \\
\hline K edema & $42.9 \%$ & $29.4 \%$ & 0.367 \\
\hline Hypopyon & $17.9 \%$ & $0.0 \%$ & 0.140 \\
\hline PS & $35.7 \%$ & $0.0 \%$ & $0.007^{\star}$ \\
\hline Iris atrophy & $0.0 \%$ & $41.2 \%$ & $<0.001$ \\
\hline Pseudophakia & $7.1 \%$ & $35.3 \%$ & $0.03{ }^{*}$ \\
\hline
\end{tabular}

IOP: intraocular pressure, KP: keratic precipitates, PS: posterior synechiae, PCR: polymerase chain reaction

\section{Discussion}


The etiologies of AU vary with differences in geographical, environmental, and genetic factors, as well as the times [18]. Moreover, changes in the incidence of familiar diseases and increased availability of diagnostic testing have altered the epidemiology of uveitis in recent years [19]. The PCR tests have revised our knowledge about the etiologies of anterior uveitis, especially hypertensive uveitis [20-23]. A 2004 report in Iran assessed idiopathic (40.7\%), HLA-B27-associated (21.5\%), FHI (17.2\%), JIA (4.8\%), and herpetic (3.8\%) AU [4]. A Tunisia report in 2007 assessed idiopathic AU (35.5\%), herpetic AU (33.7\%), FHI (8.4\%), HLA-B27-associated acute AU (7.8\%), and Behcet's disease (3.0\%) [24]. A 2012 Turkey report assessed HLA-B27-associated (14.6\%), FHI (14.6\%), herpetic AU (13.3\%), and Behcet's disease (6.6\%) [8]. A Taiwan 10-year retrospective report in 2017 showed HLA-B27-associated (38.8\%), idiopathic (25.4\%), PSS (10.9\%), and herpetic AU (10.1\%) [7]. In addition to idiopathic and HLA-B27-associated acute AU, our study demonstrated that herpetic $\mathrm{AU}$ accounted for a higher percentage than that reported in previous studies, which emphasizes the more important role of modern PCR tests in the etiology diagnosis of AU.

Most AU patients had unilateral disease (83.7\%), and initial bilateral presentations were more often seen in intermediate uveitis, posterior uveitis, and panuveitis in a Taiwanese report [7]. Our study demonstrated similar results, with $87.7 \%$ of $A U$ patients presenting with unilateral eye disease.

HLA-B27 is the best-known immune biomarker of AU. The seropositivity rate, different in several ethnicities, was reported to be $7.7 \%$ in a healthy Taiwanese population [25]. HLA-B27-associated acute AU has distinct characteristics in AU. Approximately $50 \%-75 \%$ of patients with HLA-B27-associated acute AU have seronegative spondyloarthropathy, with ankylosing spondylitis (AS) being the most common diagnosis [26]. A Chinese report indicated that the prevalence of AAU in AS was 15.8\%, and the presence of AAU in AS patients may be associated with higher disease activity, poor functional ability, and advanced physical impairment [27]. Conversely, acute AU is the most common ocular manifestation in HLA-B27 positive seronegative spondyloarthropathies [28]. HLA-B27-associated AU classically presents as an acute $\mathrm{AU}$ with symptomatic, unilateral, sudden-onset, and limited-duration anterior segment inflammation [29]. The first attack of HLA-B27-associated acute AU occurs between 20 and 40 years of age in the majority of cases, approximately 10 years younger than that observed in patients with HLAB27-negative AU [30]. Male preponderance was noted, with men affected 1.5-2.5 times more frequently than females [31, 32]. In this study, 28 patients with HLA-B27-associated acute AU exhibited a mean age of 38.8 years, an acute course of $96.4 \%$ with a mean symptom duration of only 9.6 days, but only $46.4 \%$ of occurrences in males (Table 3).

Ocular hypertension is a specific sign of active uveitis, especially in non-HLA-B27-associated acute AU. Increased availability of PCR enhances the diagnosis of hypertensive AU to detect herpetic etiologies and even connect herpetic AU to previously presumed PSS or FHI. For example, both CMV and the Rubella virus have been implicated as etiologies of PSS or FHI [21-23, 33]. In the current study, ocular hypertension accounted for $26.8 \%$ (30/112, Table 2$)$ of all cases. Approximately $30.0 \%(9 / 30)$ of ocular hypertension demonstrated positive in PCR test and all were CMV. A Thailand study defined ocular hypertension as IOP $>25 \mathrm{mmHg}$ and demonstrated $32 \%$ positivity in herpesviridae PCR, including $19 \%$ for CMV, 10\% for HSV, and 3\% for VZV [34]. In our study, 27 cases had IOP > 25mmHg and $25.9 \%(7 / 27)$ were 
positive for herpesviridae PCR with all CMV infections. A high percentage of CMV AU was found in our retrospective review and confirmed in this prospective study [17]. CMV seroprevalence tended to be the highest in South America, Africa and Asia and lowest in Western Europe and the United States [35]. This may reflect the high CMV carrier rate in Asia. The characteristics of CMV AU were reported in a study: mean age, 55.5 years, mean IOP, $29.2 \mathrm{mmHg}$; KP in $91.4 \%$; and iris atrophy $25.7 \%$ [36]. Our study revealed similar characteristics: mean age, 59.2 years, mean IOP, $26.3 \mathrm{mmHg}, \mathrm{KP} 88.2 \%$; and iris atrophy $41.2 \%$ (Table 3).

In addition to an idiopathic etiology, HLA-B27-associated acute AU and CMV AU were the two most popular specific diseases. The comparison between these two etiologies of AU was clarified in this study (Table 3). If patients with AU present with younger age, lower IOP than the fellow eye, first episode, acute course, shorter symptom duration, more PS, less iris atrophy, HLA-B27-associated acute AU is a preferred etiology compared to CMV. For AU patients with ocular hypertension, PCR analysis of the anterior chamber fluid is important to detect herpetic etiology.

Our previous study retrospectively collected the aqueous PCR data of HLA-B27 negative AU patients with increased IOP or corneal edema [17]. In that retrospective study, $41.1 \%$ of the enrolled patients were herpesviridae-positive. Compared with the current prospective study, $29.6 \%$ of the patients were herpesviridae-positive, and all of them were CMV. The CMV-AU group (Table 3) showed older age, more iris atrophy, and more pseudophakia. Both studies indicated that a significant proportion of patients with herpesviridae or CMV-positive AU had undergone intraocular lens implantation.

This study had three major limitations. First, the number of patients was small. Second, some patients received first aid at local clinics or other facilities, which may have affected ocular presentation. The treatment details, such as corticosteroids, IOP-controlling agents, and mydriatics, were not recorded. Third, this study only enrolled patients from tertiary referral centers, which could not represent the overall epidemiology. However, this study provides readers with guidelines for the care of patients with AU. First, the protocol mentioned in the Methods section can be used in clinical practice. Among several clinical parameters, IOP has exhibited strong evidence for differential diagnoses. Second, PCR is a useful tool for identifying herpetic etiology, especially in cases of ocular hypertension.

In conclusion, this prospective study identified the epidemiological and clinical features of anterior uveitis in southern Taiwan. The leading etiologies of AU were idiopathic acute, HLA-B27-associated, and CMV AU. The PCR test is an important adjunct.

\section{List Of Abbreviations}

AU: anterior uveitis, HLA: human leukocyte antigen, PSS: Posner-Schlossman syndrome, FHI: Fuchs heterochromic iridocyclitis, JIA: juvenile idiopathic uveitis, HSV: herpes simplex virus, VZV: varicella-zoster virus, CMV: cytomegalovirus, EBV: Epstein-Barr virus, PCR: polymerase chain reaction, IOP: intraocular pressure, KP: keratic precipitates, PS: posterior synechiae, SD: standard deviation, AS: ankylosing spondylitis 


\section{Declarations}

\section{Availability of data and materials}

The datasets used and/or analyzed during the current study are available from the corresponding author on reasonable request.

\section{Competing interests}

The authors declare that they have no competing interests.

\section{Funding}

This work was supported by grant CMRPG8H1281 from the Kaohsiung Chang Gung Memorial Hospital, Taiwan.

\section{Acknowledgements}

The manuscript was supported by the Kaohsiung Chang Gung Memorial Hospital, Taiwan (CMRPG8H1281).

\section{Competing Interests}

The authors have no relevant financial or non-financial interests to disclose

\section{Author Contributions}

All authors have participated directly in planning and execution of the work and have approved the final version of the manuscript. WYC and SCC: acquisition and analysis of data, drafting and writing the manuscript; SJS and HKK: participated in the design of the study, analysis of data. All authors have read and approved the final manuscript.

\section{Ethics approval}

The study adhered to the Declaration of Helsinki and was approved by the Institutional Review Board of the Chang Gung Memorial Hospital (study reference number: 201702154A3).

\section{Consent to participate}

All participants provided their written informed consent to participate in the study.

\section{Consent for publication}

Not applicable.

\section{References}


1. Miserocchi E, Fogliato G, Modorati G, Bandello F (2013) Review on the worldwide epidemiology of uveitis. Eur J Ophthalmol 23(5):705-717. https://doi: 10.5301/ejo.5000278.

2. de Smet MD, Taylor Sr, Bodaghi B, Miserocchi E, Murray Pi, Pleyer U, Zierhut M, Barisani-Asenbauer T, LeHoang P, Lightman S (2011) Understanding uveitis: the impact of research on visual outcomes. Prog Retin Eye Res 30(6):452-470. https://doi: 10.1016/j.preteyeres.2011.06.005.

3. Suttorp-Schulten MS, Rothova A (1996) The possible impact of uveitis in blindness: a literature survey. Br J Ophthalmol 80(9):844-848. https://doi: 10.1136/bjo.80.9.844.

4. Soheilian M, Heidari K, Yazdani S, Shahsavari M, Ahmadieh H, Dehghan M (2004) Patterns of uveitis in a tertiary eye care center in Iran. Ocul Immunol Inflamm 12(4):297-310. https://doi: $10.1080 / 092739490500174$.

5. Rao NA (2013) Uveitis in developing countries. Indian J Ophthalmol 61(6):253-254. https://doi: 10.4103/0301-4738.114090.

6. Jabs DA, Busingye $J$ (2013) Approach to the diagnosis of the uveitides. Am J Ophthalmol 156(2):228-236. https://doi: 10.1016/j.ajo.2013.03.027.

7. Chen SC, Chuang CT, Chu MY, Sheu SJ (2017) Patterns and Etiologies of Uveitis at a Tertiary Referral Center in Taiwan. Ocul Immunol Inflamm 25:S31-38. https://doi: 10.1080/09273948.2016.1189577.

8. Guney E, Akcay Bi, Erdogan G, Unlu C, Akcali G, Bayramlar H (2012) The etiological features of anterior uveitis in a Turkish population. Clin Ophthalmol 6:845-849. https://doi: 10.2147/OPTH.S32271.

9. Hwang DK, Chou Yj, Pu Cy, Chou P (2012) Epidemiology of uveitis among the Chinese population in Taiwan: a population-based study. Ophthalmology 119(11):2371-2376. https://doi: 10.1016/j.ophtha.2012.05.026.

10. Llorenç V, Mesquida M, Sainz de la Maza M, Keller J, Molins B, Espinosa G, Hernandez MV, GonzalezMartín J, Adán A (2015) Epidemiology of uveitis in a Western urban multiethnic population. The challenge of globalization. Acta Ophthalmol 93(6):561-567. https://doi: 10.1111/aos.12675.

11. Grajewski RS, Caramoy A, Frank KF, Rubbert-Roth A, Fätkenheuer G, Kirchhof B, Cursiefen C, Heindl LM (2015) Spectrum of Uveitis in A German Tertiary Center: Review of 474 Consecutive Patients. Ocul Immunol Inflamm 23(4):346-352. https://doi: 10.3109/09273948.2014.1002567.

12. Jakob E, Reuland Ms, Mackensen F, Harsch N, Fleckenstein M, Lorenz Hm, Max R, Becker MD (2009) Uveitis subtypes in a german interdisciplinary uveitis center-analysis of 1916 patients. J Rheumatol 36(1):127-136. https://doi: 10.3899/jrheum.080102.

13. Jones NP (2015) The Manchester Uveitis Clinic: the first 3000 patients--epidemiology and casemix. Ocul Immunol Inflamm 23(2):118-126. https://doi: 10.3109/09273948.2013.855799.

14. Agrawal RV, Murthy S, Sangwan V, Biswas J (2010) Current approach in diagnosis and management of anterior uveitis. Indian J Ophthalmol 58(1):11-19. https://doi: 10.4103/0301-4738.58468.

15. Gueudry J, Muraine M (2018) Anterior uveitis. J Fr Ophtalmol 41(2):170-182. https://doi: 10.1016/j.jfo.2017.06.012. 
16. Chang JH, McCluskey Pj, Wakefield D (2005) Acute anterior uveitis and HLA-B27. Surv Ophthalmol 50(4):364-388. https://doi: 10.1016/j.survophthal.2005.04.003.

17. Hsiao YT, Kuo MT, Chiang WY, Chao TL, Kuo HK (2019) Epidemiology and clinical features of viral anterior uveitis in southern Taiwan-diagnosis with polymerase chain reaction. BMC Ophthalmol 19(1):87. https://doi: 10.1186/s12886-019-1093-2.

18. Chang JH, Wakefield D (2002) Uveitis: a global perspective. Ocul Immunol Inflamm 10(4):263-279. https://doi: 10.1076/ocii.10.4.263.15592.

19. Tsirouki T, Dastiridou A, Symeonidis C, Tounakaki O, Brazitikou I, Kalogeropoulos C, Androudi S (2018) A Focus on the Epidemiology of Uveitis. Ocul Immunol Inflamm 26(1):2-16. https://doi: 10.1080/09273948.2016.1196713.

20. De Groot-Mijnes JD, Rothova A, Van Loon Am, Schuller M, Ten Dam-Van Loon Nh, De Boer Jh, Schuurman R, Weersink AJ (2006) Polymerase chain reaction and Goldmann-Witmer coefficient analysis are complimentary for the diagnosis of infectious uveitis. Am J Ophthalmol 141(2):313-318. https://doi: 10.1016/j.ajo.2005.09.017.

21. Teoh Sb, Thean L, Koay E (2005) Cytomegalovirus in aetiology of Posner-Schlossman syndrome: evidence from quantitative polymerase chain reaction. Eye (Lond) 19(12):1338-1340. https://doi: $10.1038 /$ sj.eye.6701757.

22. Chee SP, Jap A (2008) Presumed fuchs heterochromic iridocyclitis and Posner-Schlossman syndrome: comparison of cytomegalovirus-positive and negative eyes. Am J Ophthalmol 146(6):883889. https://doi: 10.1016/j.ajo.2008.09.001.

23. Hassan R, White Lr, Stefanoff Cg, de Oliveira De, Felisbino Fe, Klumb Ce, Bacchi Ce, Seuánez Hn, Zalcberg IR (2006) Epstein-Barr virus (EBV) detection and typing by PCR: a contribution to diagnostic screening of EBV-positive Burkitt's lymphoma. Diagn Pathol 1(17). https://doi: 10.1186/1746-1596-117.

24. Khairallah M, Yahia Sb, Ladjimi A, Messaoud R, Zaouali S, Attia S, Jenzeri S, Jelliti B (2007) Pattern of uveitis in a referral centre in Tunisia, North Africa. Eye (Lond) 21(1):33-39. https://doi: $10.1038 /$ sj.eye.6702111.

25. Wei JC, Sung-Ching HW, Hsu YW, Wen YF, Wang WC, Wong RH, Lu HF, Gaalen FA, Chang WC (2005) Interaction between HLA-B60 and HLA-B27 as a Better Predictor of Ankylosing Spondylitis in a Taiwanese Population. PLoS One 10(10):e0137189. https://doi: 10.1371/journal.pone.0137189.

26. Wakefield D, Yates W, Amjadi S, McCluskey P (2016) HLA-B27 Anterior Uveitis: Immunology and Immunopathology. Ocul Immunol Inflamm 24(4):450-459. https://doi: 10.3109/09273948.2016.1158283.

27. Chen CH, Lin Kc, Chen Ha, Liao Ht, Liang Th, Wang Hp, Chou CT (2007) Association of acute anterior uveitis with disease activity, functional ability and physical mobility in patients with ankylosing spondylitis: a cross-sectional study of Chinese patients in Taiwan. Clin Rheumatol 26(6):953-957. https://doi: 10.1007/s10067-006-0403-2. 
28. Jhaj G, Kopplin LJ (2018) Ocular features of the HLA-B27-positive seronegative spondyloarthropathies. Curr Opin Ophthalmol 29(6):552-557. https://doi: 10.1097/ICU.0000000000000525.

29. Kopplin LJ, Mount G, Suhler EB (2016) Review for Disease of the Year: Epidemiology of HLA-B27 Associated Ocular Disorders. Ocul Immunol Inflamm 24(4):470-475. https://doi: 10.1080/09273948.2016.1175642.

30. Wakefield D, Chang Jh, Amjadi S, Maconochie Z, Abu El-Asrar A, McCluskey P (2011) What is new HLA-B27 acute anterior uveitis? Ocul Immunol Inflamm 19(2):139-144. https://doi: 10.3109/09273948.2010.542269.

31. Linssen A, Meenken C (1995) Outcomes of HLA-B27-positive and HLA-B27-negative acute anterior uveitis. Am J Ophthalmol 120(3):351-361. https://doi: 10.1016/s0002-9394(14)72165-8.

32. Tay-Kearney ML, Schwam BI, Lowder C, Dunn Jp, Meisler Dm, Vitale S, Jabs DA (1996) Clinical features and associated systemic diseases of HLA-B27 uveitis. Am J Ophthalmol 121(1):47-56. https://doi: 10.1016/s0002-9394(14)70533-1.

33. Kongyai N, Sirirungsi W, Pathanapitoon K, Tananuvat N, Kunavisarut P, Leechanachai P, de GrootMijnes Jd, Rothova A (2012) Viral causes of unexplained anterior uveitis in Thailand. Eye (Lond) 26(4):529-534. https://doi: 10.1038/eye.2011.363.

34. Khieu C, Kongyai N, Pathanapitoon K, Van Der Eijk AA, Rothova A (2020) Causes of Hypertensive Anterior Uveitis in Thailand. Ocul Immunol Inflamm 28(4):559-565. https://doi: 10.1080/09273948.2019.1678651.

35. Cannon MJ, Schmid Ds, Hyde TB (2010) Review of cytomegalovirus seroprevalence and demographic characteristics associated with infection. Rev Med Virol 20(4):202-213. https://doi: 10.1002/rmv.655.

36. Touhami S, Qu L, Angi M, Bojanova M, Touitou V, Lehoang P, Rozenberg F, Bodaghi B (2018) Cytomegalovirus Anterior Uveitis: Clinical Characteristics and Long-term Outcomes in a French Series. Am J Ophthalmol 194:134-142. https://doi: 10.1016/j.ajo.2018.07.021. 